\title{
On Pillai's problem with Pell numbers and powers of 2
}

\author{
Mohand Ouamar Hernane, Florian Luca, Salah Eddine Rihane and Alain Togbé
}

To the memory of S. Srinivasan

\begin{abstract}
In this paper, we find all integers $c$ having at least two representations as a difference between a Pell number and a power of 2 .

Keywords. Pell numbers, Linear form in logarithms, reduction method.

2010 Mathematics Subject Classification. primary 11B39; secondary 11J86.
\end{abstract}

\section{Introduction}

It is well-known that the sequence $\left\{P_{n}\right\}_{n \geq 0}$ of Pell numbers is defined by

$$
P_{0}=0, \quad P_{1}=1, \quad P_{n+2}=2 P_{n+1}+P_{n}, \quad n \geq 0 .
$$

The first Pell numbers are

$$
0,1,2,5,12,29,70,169,408,985,2378,5741,13860, \ldots
$$

In this paper, we are interested in the Diophantine equation

$$
P_{n}-2^{m}=c
$$

for a fixed $c$ and variable $m$ and $n$. In particular, we are interested in those integers $c$ admitting at least two representations as a difference between a Pell number and a power of 2 . This is a variation of the equation

$$
a^{x}-b^{y}=c,
$$

in non-negative integers $(x, y)$ where $a, b, c$ are given fixed positive integers. The history of equation $(1 . .2)$ is very rich and goes back to 1935 when Herschfeld [He35], [He36] studied the particular case $(a, b)=(2,3)$. Extending Herschfeld's work, Pillai [Pi36], [Pi37] proved that if $a, b$ are coprime positive integers then there exists $c_{0}(a, b)$ such that if $c>c_{0}(a, b)$ is an integer, then equation (1..2) has at most one positive integer solution $(x, y)$. Since then, variations of equation (1..2) has been intensively studied. Some recent results related to equation (1..1) are obtained by the third author and his collaborators in which they replaced Pell numbers $P_{n}$ by the Fibonacci numbers $F_{n}$ (see [DdLuR17]), Tribonacci numbers (see [BrLuYa17]), and $k$-generalized Fibonacci numbers (see [DdGLu17]).

The aim of this paper is to prove the following result.

Theorem 1.1. The only integers $c$ having at least two representations of the form $P_{n}-2^{m}$ are $c \in\{-4,-3,-2,-1,0,1,4\}$. Furthermore, all the representations of the above integers as $P_{n}-2^{m}$ with integers $n \geq 0$ and $m \geq 0$ are given by

$$
\begin{aligned}
-4 & =P_{4}-2^{4}=P_{0}-2^{2} \\
-3 & =P_{5}-2^{5}=P_{3}-2^{3}=P_{1}-2^{1} \\
-2 & =P_{2}-2^{2}=P_{0}-2^{1} \\
-1 & =P_{1}-2^{1}=P_{0}-2^{0} \\
0 & =P_{2}-2^{1}=P_{1}-2^{0} \\
1 & =P_{3}-2^{2}=P_{2}-2^{0} \\
4 & =P_{4}-2^{3}=P_{3}-2^{0} .
\end{aligned}
$$

We thank episciences.org for providing open access hosting of the electronic journal Hardy-Ramanujan Journal 
We organize this paper as follows. In Section 2., we recall some results useful for the proof of Theorem 1.1. The proof of Theorem 1.1 is done in the last section.

\section{Auxiliary results}

The next tools are related to the transcendental approach to solve Diophantine equations. For a nonzero algebraic number $\gamma$ of degree $d$ over $\mathbb{Q}$, whose minimal polynomial over $\mathbb{Z}$ is $a \prod_{j=1}^{d}\left(X-\gamma^{(j)}\right)$, we denote by

$$
h(\gamma)=\frac{1}{d}\left(\log |a|+\sum_{j=1}^{d} \log \max \left(1,\left|\gamma^{(j)}\right|\right)\right)
$$

the usual absolute logarithmic height of $\gamma$.

Lemma 2.1. Let $\gamma_{1}, \ldots, \gamma_{s}$ be a real algebraic numbers and let $b_{1}, \ldots, b_{s}$ be nonzero rational integer numbers. Let $D$ be the degree of the number field $\mathbb{Q}\left(\gamma_{1}, \ldots, \gamma_{s}\right)$ over $\mathbb{Q}$ and let $A_{j}$ be a positive real number satisfying

$$
A_{j}=\max \left\{D h\left(\gamma_{j}\right),\left|\log \gamma_{j}\right|, 0.16\right\} \text { for } j=1, \ldots, s .
$$

Assume that

$$
B \geq \max \left\{\left|b_{1}\right|, \ldots,\left|b_{s}\right|\right\} .
$$

If $\gamma_{1}^{b_{1}} \cdots \gamma_{s}^{b_{s}} \neq 1$, then

$$
\left|\gamma_{1}^{b_{1}} \cdots \gamma_{s}^{b_{s}}-1\right| \geq \exp \left(-C(s, D)(1+\log B) A_{1} \cdots A_{s}\right),
$$

where $C(s, D):=1.4 \cdot 30^{s+3} \cdot s^{4.5} \cdot D^{2}(1+\log D)$.

Lemma 2.2. Assume that $\tau$ and $\mu$ are real numbers and $M$ is a positive integer. Let $p / q$ be the convergent of the continued fraction of the irrational $\tau$ such that $q>6 M$, and let $A, B, \mu$ be some real numbers with $A>0$ and $B>1$. Let $\varepsilon=\|\mu q\|-M \cdot\|\tau q\|$, where $\|\cdot\|$ denotes the distance from the nearest integer. If $\varepsilon>0$, then there is no solution of the inequality

$$
0<m \tau-n+\mu<A B^{-k}
$$

in positive integers $m, n$ and $k$ with

$$
m \leq M \quad \text { and } \quad k \geq \frac{\log (A q / \varepsilon)}{\log B} .
$$

\section{Proof of Theorem 1.1}

Assume that there exist positive integers $n, m, n_{1}, m_{1}$ such that $(n, m) \neq\left(n_{1}, m_{1}\right)$, and

$$
P_{n}-2^{m}=P_{n_{1}}-2^{m_{1}} .
$$

Because of the symmetry, we can assume that $m \geq m_{1}$. If $m=m_{1}$, then $P_{n}=P_{n_{1}}$, so $(n, m)=$ $\left(n_{1}, m_{1}\right)$, contradicting our assumption. Thus, $m>m_{1}$. Since

$$
P_{n}-P_{n_{1}}=2^{m}-2^{m_{1}},
$$

and the right-hand side is positive, we get that the left-hand side is also positive and so $n>n_{1}$. Thus, $n \geq 2$ and $n_{1} \geq 1$. We use the Binet formula

$$
P_{k}=\frac{\alpha^{k}-\beta^{k}}{2 \sqrt{2}} \text { for all } k \geq 0,
$$


where $(\alpha, \beta):=(1+\sqrt{2}, 1-\sqrt{2})$ are the roots of the characteristic equation of the Pell sequence. It is well-known that

$$
\alpha^{k-2} \leq P_{k} \leq \alpha^{k-1} \quad \text { for all } k \geq 1
$$

Using the equation (3..1), we get

$$
\begin{aligned}
& \alpha^{n-4} \leq P_{n-2} \leq P_{n}-P_{n_{1}}=2^{m}-2^{m_{1}}<2^{m}, \\
& \alpha^{n-1} \geq P_{n}>P_{n}-P_{n_{1}}=2^{m}-2^{m_{1}} \geq 2^{m-1},
\end{aligned}
$$

therefore

$$
1+\left(\frac{\log 2}{\log \alpha}\right)(m-1)<n<\left(\frac{\log 2}{\log \alpha}\right) m+4
$$

If $n<150$, then $m \leq 200$. We ran a computer program for $2 \leq n_{1}<n \leq 150$ and $1 \leq m_{1}<m<200$ and found only the solutions from list (1..3). From now, we assume that $n \geq 150$.

Note that the inequality (3..3) implies that $\frac{m}{2}<n$. So, to solve equation (3..1), we need an upper bound for $n$.

\section{A. Bounding $n$}

Using the Binet formula in the Diophantine equation (3..1), we get

$$
\begin{aligned}
\left|\frac{\alpha^{n}}{2 \sqrt{2}}-2^{m}\right| & =\left|\frac{\beta^{n}}{2 \sqrt{2}}+\frac{\alpha^{n_{1}}-\beta^{n_{1}}}{2 \sqrt{2}}-2^{m_{1}}\right| \leq \frac{\alpha^{n_{1}}+2}{2 \sqrt{2}}+2^{m_{1}} \\
& \leq \frac{\alpha^{n_{1}}}{\sqrt{2}}+2^{m_{1}}<2 \max \left\{\alpha^{n_{1}}, 2^{m_{1}}\right\} .
\end{aligned}
$$

Multiplying by $2^{-m}$, using the relation (3..2a) and using the fact that $2<\alpha$, we get

$$
\left|(\sqrt{2})^{-1} \alpha^{n} 2^{-m-1}-1\right|<2 \max \left\{\frac{\alpha^{n_{1}}}{2^{m}}, 2^{m_{1}-m}\right\}<\max \left\{\alpha^{n_{1}-n+5}, 2^{m_{1}-m+1}\right\} .
$$

For the left-hand side, we apply Theorem 2.1 with the data

$$
s=3, \quad \gamma_{1}=\sqrt{2}, \quad \gamma_{2}=\alpha, \quad \gamma_{3}=2, \quad b_{1}=-1, \quad b_{2}=n, \quad b_{3}=-m-1 .
$$

Throughout we work with $\mathbb{K}:=\mathbb{Q}(\sqrt{2})$ with $D=2$. Since $\max \{1, n, m+1\} \leq 2 n$ we take $B:=2 n$. Furthermore, we take $A_{1}:=2 h\left(\gamma_{1}\right)=\log 2, A_{2}:=2 h\left(\gamma_{2}\right)=\log \alpha, A_{3}:=2 h\left(\gamma_{3}\right)=2 \log 2$. Put

$$
\Lambda=(\sqrt{2})^{-1} \alpha^{n} 2^{-m-1}-1 .
$$

If $\Lambda=0$, then $\alpha^{2 n} \in \mathbb{Q}$, which is false. Thus, $\Lambda \neq 0$. Then, by Theorem 2.1, the left-hand side of (3..4) is bounded as

$$
\log |\Lambda|>-1.4 \cdot 30^{6} \cdot 3^{4.5} \cdot 2^{2}(1+\log 2)(1+\log 2 n)(\log 2)(\log \alpha)(2 \log 2) .
$$

Comparing with (3..4), we get

$$
\min \left\{\left(n-n_{1}-5\right) \log \alpha,\left(m-m_{1}-1\right) \log 2\right\}<8.3 \times 10^{11}(1+\log 2 n),
$$

which gives

$$
\min \left\{\left(n-n_{1}\right) \log \alpha,\left(m-m_{1}\right) \log 2\right\}<8.4 \times 10^{11}(1+\log 2 n) .
$$

Now the argument splits into two cases.

Case 1. $\min \left\{\left(n-n_{1}\right) \log \alpha,\left(m-m_{1}\right) \log 2\right\}=\left(n-n_{1}\right) \log \alpha$. 
In this case, we rewrite (3..1) as

$$
\left|\left(\frac{\alpha^{n-n_{1}}-1}{2 \sqrt{2}}\right) \alpha^{n_{1}}-2^{m}\right|=\left|\frac{\beta^{n}-\beta^{n_{1}}}{2 \sqrt{2}}-2^{m_{1}}\right|<2^{m_{1}}+1 \leq 2^{m_{1}+1},
$$

which implies

$$
\left|\left(\frac{\alpha^{n-n_{1}}-1}{2 \sqrt{2}}\right) \alpha^{n_{1}} 2^{-m}-1\right|<2^{m_{1}-m+1} .
$$

We put

$$
\Lambda_{1}=\left(\frac{\alpha^{n-n_{1}}-1}{2 \sqrt{2}}\right) \alpha^{n_{1}} 2^{-m}-1
$$

Clearly, $\Lambda_{1} \neq 0$, for if $\Lambda_{1}=0$, then

$$
\left(\alpha^{n-n_{1}}-1\right) \alpha^{n_{1}}=\sqrt{2} \times 2^{m} .
$$

Conjugating this relation in $\mathbb{K}$, we get that

$$
\left(\alpha^{n-n_{1}}-1\right) \alpha^{n_{1}}=-\left(\beta^{n-n_{1}}-1\right) \beta^{n_{1}} .
$$

The absolute value of the left-hand side is at least $\alpha^{n}-\alpha^{n_{1}} \geq \alpha^{n-2} \geq \alpha^{188}$, while the absolute value of the right-hand side is at most $\left(|\beta|^{n-n_{1}}+1\right)|\beta|^{n_{1}}<2$, which is a contradiction.

We apply Theorem 2.1 by taking $s=3$, and

$$
\gamma_{1}=\frac{\alpha^{n-n_{1}}-1}{2 \sqrt{2}}, \quad \gamma_{2}=\alpha, \quad \gamma_{3}=2, \quad b_{1}=1, \quad b_{2}=n_{1}, \quad b_{3}=-m
$$

The minimal polynomial of $\gamma_{1}$ divides

$$
8 x^{2}-8 P_{n-n_{1}} x-\left((-1)^{n-n_{1}}+1-Q_{n-n_{1}}\right),
$$

where $\left\{Q_{k}\right\}_{k \geq 0}$ is the Pell-Lucas companion sequence of the Pell sequence given by $Q_{0}=2, Q_{1}=1$, $Q_{k+2}=2 Q_{k+1}+Q_{k}$ for all $k \geq 0$, for which the Binet formula of its general term is

$$
Q_{k}=\alpha^{k}+\beta^{k} \quad \text { for all } k \geq 0 .
$$

Thus, we obtain

$$
\begin{aligned}
h\left(\gamma_{1}\right) & \leq \frac{1}{2}\left(\log 8+\log \left(\frac{\alpha^{n-n_{1}}+1}{2 \sqrt{2}}\right)\right)<\frac{1}{2} \log \left(4 \sqrt{2} \alpha^{n-n_{1}}\right) \\
& <\frac{1}{2}\left(n-n_{1}+2\right) \log \alpha<4.2 \times 10^{11}(1+\log 2 n) .
\end{aligned}
$$

So, we can take $A_{1}:=8.4 \times 10^{11}(1+\log 2 n)$. Further, as before, we can take $A_{2}:=\log \alpha$ and $A_{3}:=2 \log 2$. Finally, since $\max \left\{1, n_{1}, m\right\} \leq 2 n$, we can take $B:=2 n$. We then get that

$$
\log \left|\Lambda_{1}\right|>-1.4 \cdot 30^{6} \cdot 3^{4.5} \cdot 2^{2}(1+\log 2)(1+\log 2 n) \times\left(8.4 \times 10^{11}(1+\log n)\right)(\log \alpha)(2 \log 2) .
$$

Thus,

$$
\log \left|\Lambda_{1}\right|>-1.1 \cdot 10^{24}(1+\log 2 n)^{2} .
$$

Comparing this with (3..5), we get that

$$
\left(m-m_{1}\right) \log 2<1.1 \cdot 10^{24}(1+\log 2 n)^{2} .
$$

Case 2. $\min \left\{\left(n-n_{1}\right) \log \alpha,\left(m-m_{1}\right) \log 2\right\}=\left(m-m_{1}\right) \log 2$. 
In this case, we rewrite (3..1) as

$$
\left|\frac{\alpha^{n}}{2 \sqrt{2}}-2^{m_{1}}\left(2^{m-m_{1}}-1\right)\right|=\left|\frac{\beta^{n}+\alpha^{n_{1}}-\beta^{n_{1}}}{2 \sqrt{2}}\right|<\frac{\alpha^{n_{1}}+2}{2 \sqrt{2}}<\alpha^{n_{1}},
$$

so

$$
\begin{aligned}
\left|\left(\sqrt{2}\left(2^{m-m_{1}}-1\right)\right)^{-1} \alpha^{n} 2^{-m_{1}-1}-1\right| & <\frac{\alpha^{n_{1}}}{2^{m}-2^{m_{1}}} \leq \frac{2 \alpha^{n_{1}}}{2^{m}} \\
& \leq 2 \alpha^{n_{1}-n+4}<\alpha^{n_{1}-n+5} .
\end{aligned}
$$

Let

$$
\Lambda_{2}=\left(\sqrt{2}\left(2^{m-m_{1}}-1\right)\right)^{-1} \alpha^{n} 2^{-m_{1}-1}-1 .
$$

Clearly, $\Lambda_{2} \neq 0$, for if $\Lambda_{2}=0$ implies $\alpha^{2 n} \in \mathbb{Q}$, which is not possible.

We apply again Theorem 2.1. In this application, we take again $s=3$, and

$$
\gamma_{1}=\sqrt{2}\left(2^{m-m_{1}}-1\right), \quad \gamma_{2}=\alpha, \quad \gamma_{3}=2, \quad b_{1}=-1, \quad b_{2}=n, \quad b_{3}=-m_{1}-1 .
$$

The minimal polynomial of $\gamma_{1}$ is $x^{2}-2\left(2^{m-m_{1}}-1\right)^{2}$. Thus

$$
h\left(\gamma_{1}\right)=\log \left(\sqrt{2}\left(2^{m-m_{1}}-1\right)\right)<\left(m-m_{1}+1\right) \log 2<8.5 \times 10^{11}(1+\log 2 n) .
$$

So, we can take $A_{1}:=1.7 \times 10^{12}(1+\log 2 n)$. Further, as before, we can take $A_{2}:=\log \alpha$ and $A_{3}:=2 \log 2$. Finally, since $\max \left\{1, n, m_{1}+1\right\} \leq 2 n$, we can take $B:=2 n$.

We then get that

$$
\log \left|\Lambda_{2}\right|>-1.4 \cdot 30^{6} \cdot 3^{4.5} \cdot 2^{2}(1+\log 2)(1+\log 2 n) \times\left(1.7 \times 10^{12}(1+\log 2 n)\right)(\log \alpha)(2 \log 2) .
$$

Thus,

$$
\log \left|\Lambda_{1}\right|>-2.1 \cdot 10^{24}(1+\log 2 n)^{2} .
$$

Comparing this with (3..8), we get that

$$
\left(n-n_{1}\right) \log \alpha<2.2 \cdot 10^{24}(1+\log 2 n)^{2} .
$$

Thus, in both Case 1 and Case 2, we have

$$
\begin{aligned}
\min \left\{\left(n-n_{1}\right) \log \alpha,\left(m-m_{1}\right) \log 2\right\} & <8.4 \cdot 10^{11}(1+\log 2 n) \\
\max \left\{\left(n-n_{1}\right) \log \alpha,\left(m-m_{1}\right) \log 2\right\} & <2.2 \cdot 10^{24}(1+\log 2 n)^{2} .
\end{aligned}
$$

We now finally rewrite equation (3..1) as

$$
\left|\frac{\left(\alpha^{n-n_{1}}-1\right)}{2 \sqrt{2}} \alpha^{n_{1}}-2^{m_{1}}\left(2^{m-m_{1}}-1\right)\right|=\left|\frac{\beta^{n}-\beta^{n_{1}}}{2 \sqrt{2}}\right|<|\beta|^{n_{1}}=\frac{1}{\alpha^{n_{1}}} .
$$

Dividing both sides by $2^{m}-2^{m_{1}}$, we get

$$
\begin{aligned}
\left|\left(\frac{\alpha^{n-n_{1}}-1}{\sqrt{2}\left(2^{m-m_{1}}-1\right)}\right) \alpha^{n_{1}} 2^{-m_{1}-1}-1\right| & <\frac{1}{\alpha^{n_{1}}\left(2^{m}-2^{m_{1}}\right)} \leq \frac{2}{\alpha^{n_{1}} 2^{m}} \\
& \leq 2 \alpha^{4-n-n_{1}} \leq \alpha^{4-n}
\end{aligned}
$$

because $\alpha^{n_{1}} \geq \alpha>2$. To find a lower-bound on the left-hand side, we use again Theorem 2.1 with $s=3$, and

$$
\gamma_{1}=\frac{\alpha^{n-n_{1}}-1}{\sqrt{2}\left(2^{m-m_{1}}-1\right)}, \quad \gamma_{2}=\alpha, \quad \gamma_{3}=2, \quad b_{1}=1, \quad b_{2}=n_{1}, \quad b_{3}=-m_{1}-1 .
$$


Using $h(x / y)=h(x)+h(y)$ for any two nonzero algebraic numbers $x$ and $y$, we have

$$
\begin{aligned}
h\left(\gamma_{1}\right) & \leq h\left(\frac{\alpha^{n-n_{1}}-1}{\sqrt{2}}\right)+h\left(2^{m-m_{1}}-1\right)<\frac{1}{2}\left(n-n_{1}+2\right) \log \alpha+\left(m-m_{1}\right) \log 2 \\
& <2.5 \cdot 10^{24}(1+\log 2 n)^{2},
\end{aligned}
$$

where in the above chain of inequalities, we used the argument from (3..7) as well as the bound (3..9). So, we can take $A_{1}:=5 \cdot 10^{24}(1+\log 2 n)^{2}$ and certainly $A_{2}:=\log \alpha$ and $A_{3}:=2 \log 2$. We need to show that if we put

$$
\Lambda_{3}=\left(\frac{\alpha^{n-n_{1}}-1}{\sqrt{2}\left(2^{m-m_{1}}-1\right)}\right) \alpha^{n_{1}} 2^{-m_{1}-1}-1,
$$

then $\Lambda_{3} \neq 0$. But $\Lambda_{3}=0$ leads to

$$
\left(\alpha^{n-n_{1}}-1\right) \alpha^{n_{1}}=\sqrt{2}\left(2^{m}-2^{m_{1}}\right),
$$

which upon conjugation in $\mathbb{K}$ leads to (3..6), which is impossible. Thus, $\Lambda_{3} \neq 0$. Theorem 2.1 gives

$$
\log \left|\Lambda_{3}\right|>-1.4 \cdot 30^{6} \cdot 3^{4.5} \cdot 2^{2}(1+\log 2)(1+\log 2 n) \times\left(5 \times 10^{24}(1+\log 2 n)^{2}\right)(\log \alpha)(2 \log 2),
$$

which together with $(3 . .10)$ gives

$$
(n-4)<6 \cdot 10^{36}(1+\log 2 n)^{3},
$$

leading to $n<7 \cdot 10^{42}$.

\section{B. Reducing $n$}

We now need to reduce the above bound for $n$ and to do so we make use of Lemma 2.2 several times. To begin with, we return to (3..4) and put

$$
\Gamma:=n \log \alpha-m \log 2-\log (2 \sqrt{2}) .
$$

For technical reasons we assume that $\min \left\{n-n_{1}, m-m_{1}\right\} \geq 20$. We go back to the inequalities for $\Lambda, \Lambda_{1}, \Lambda_{2}$. Since we assume that $\min \left\{n-n_{1}, m-m_{1}\right\} \geq 20$ we get $\left|e^{\Gamma}-1\right|=|\Lambda|<\frac{1}{4}$. Hence, $|\Lambda|<\frac{1}{2}$ and since the inequality $|x|<2\left|e^{x}-1\right|$ holds for all $x \in\left(-\frac{1}{2}, \frac{1}{2}\right)$, we get

$$
|\Gamma|<2 \max \left\{\alpha^{n_{1}-n+5}, 2^{m_{1}-m+1}\right\} \leq \max \left\{\alpha^{n_{1}-n+6}, 2^{m_{1}-m+2}\right\} .
$$

Assume $\Gamma>0$. We then have the inequality

$$
\begin{aligned}
0<n\left(\frac{\log \alpha}{\log 2}\right)-m-\frac{3}{2} & <\max \left\{\frac{\alpha^{6}}{\log 2} \alpha^{-\left(n-n_{1}\right)}, \frac{4}{\log 2} 2^{-\left(m-m_{1}\right)}\right\} \\
& <\max \left\{286 \alpha^{-\left(n-n_{1}\right)}, 6 \cdot 2^{-\left(m-m_{1}\right)}\right\} .
\end{aligned}
$$

We apply Lemma 2.2 with

$$
\tau=\frac{\log \alpha}{\log 2}, \quad \mu=-\frac{3}{2}, \quad(A, B)=(286, \alpha) \text { or }(6,2) .
$$

Let $\tau=\left[a_{0}, a_{1}, \ldots\right]=[1,3,1,2,6,1,2,11,2,2,1, \ldots]$ be the continued fraction of $\tau$. We choose $M:=$ $7 \cdot 10^{42}$ and consider the 89 -th convergent

$$
\frac{p}{q}=\frac{p_{89}}{q_{89}}=\frac{348317200801236358620935022888502708006954949997}{273930475375768068118103206105181460963472890299} .
$$


It satisfies $q=q_{89}>6 M$. Further, it yields $\varepsilon>0.49$, and therefore either

$$
n-n_{1} \leq \frac{\log (286 q / \varepsilon)}{\log \alpha}<132, \text { or } m-m_{1} \leq \frac{\log (6 q / \varepsilon)}{\log 2}<126 .
$$

In the case of $\Gamma<0$, we consider the following inequality:

$$
\begin{aligned}
m\left(\frac{\log 2}{\log \alpha}\right)-n+\frac{\log (2 \sqrt{2})}{\log \alpha} & <\max \left\{\frac{\alpha^{6}}{\log \alpha} \alpha^{-\left(n-n_{1}\right)}, \frac{4}{\log \alpha} 2^{-\left(m-m_{1}\right)}\right\} \\
& <\max \left\{225 \alpha^{-\left(n-n_{1}\right)}, 5 \cdot 2^{-\left(m-m_{1}\right)}\right\}
\end{aligned}
$$

instead and apply Lemma 2.2 with

$$
\tau=\frac{\log 2}{\log \alpha}, \quad \mu=\frac{\log (2 \sqrt{2})}{\log \alpha}, \quad(A, B)=(225, \alpha) \text { or }(5,2) .
$$

Let $\tau=\left[a_{0}, a_{1}, \ldots\right]=[0,1,3,1,2,6,1,2,11,2,2,1, \ldots]$ be the continued fraction of $\tau$ (note that the current $\tau$ is just the reciprocal of the previous $\tau$ ). Again, we choose $M=4.2 \cdot 10^{43}$, and in this case we consider the 90-th convergent

$$
\frac{p}{q}=\frac{p_{90}}{q_{90}}=\frac{116053085442077720965142600370109382756030794793}{91268753856671834783420178148248093030868562298},
$$

which satisfies $q=q_{90}>6 M$. This yields again $\varepsilon>0.49$, and therefore either

$$
n-n_{1} \leq \frac{\log (225 q / \varepsilon)}{\log \alpha}<132, \text { or } m-m_{1} \leq \frac{\log (5 q / \varepsilon)}{\log 2}<126 .
$$

These bounds agree with the bounds obtained in the case that $\Gamma>0$. As a conclusion, we have either $n-n_{1} \leq 131$ or $m-m_{1} \leq 125$ whenever $\Gamma \neq 0$.

Now, we have to distinguish between the cases $n-n_{1} \leq 131$ and $m-m_{1} \leq 125$. First, let assume that $n-n_{1} \leq 131$. In this case, we consider inequality (3..5) and assume that $m-m_{1} \geq 20$. We put

$$
\Gamma_{1}=n_{1} \log \alpha-m \log 2+\log \left(\frac{\alpha^{n-n_{1}}-1}{2 \sqrt{2}}\right) .
$$

Then inequality (3..5) implies that

$$
\left|\Gamma_{1}\right|<\frac{4}{2^{m-m_{1}}} .
$$

If we further assume that $\Gamma_{1}>0$, we then get

$$
0<n_{1}\left(\frac{\log \alpha}{\log 2}\right)-m+\frac{\log \left(\left(\alpha^{n-n_{1}}-1\right) /(2 \sqrt{2})\right.}{\log 2}<\frac{4}{(\log 2) 2^{m-m_{1}}}<\frac{6}{2^{m-m_{1}}} .
$$

Again we apply Lemma 2.2 with the same $\tau$ as in the case when $\Gamma>0$. We use the 89-th convergent $p / q=p_{89} / q_{89}$ of $\tau$ as before. But in this case we choose $(A, B):=(6,2)$ and use

$$
\mu_{k}=\frac{\log \left(\left(\alpha^{k}-1\right) /(2 \sqrt{2})\right)}{\log 2}
$$

instead of $\mu$ for each possible value of $k:=n-n_{1} \in[1,2, \ldots 131]$. We have problems at $k \in\{1,4\}$. We discard these values for now and will treat them later. For the remaining values of $k$, we get $\varepsilon>0.0004$. Hence, by Lemma 2.2, we get

$$
m-m_{1}<\frac{\log (6 q / 0.0004)}{\log 2}<172 .
$$


Thus, $n-n_{1} \leq 131$ implies $m-m_{1} \leq 171$, unless $n-n_{1} \in\{1,4\}$. A similar conclusion is reached if $\Gamma_{1}<0$ with the same two exceptions for $n-n_{1} \in\{1,4\}$. The reason we have a problem at $k \in\{1,4\}$ is because

$$
\frac{\alpha-1}{2 \sqrt{2}}=\frac{1}{2} \quad \text { and } \quad \frac{\alpha^{4}-1}{2 \sqrt{2}}=2 \alpha^{2} .
$$

So, $\Gamma_{1}=n_{1} \log \alpha-(m+1) \log 2$, or $\left(n_{1}+2\right) \log \alpha-(m-1) \log 2$ when $k=1,4$ respectively. Thus we get that

$$
\left|\tau-\frac{m+1}{n_{1}}\right|<\frac{4}{2^{m-m_{1}} n_{1}}, \quad \text { or } \quad\left|\tau-\frac{m-1}{n_{1}+2}\right|<\frac{4}{2^{m-m_{1}}\left(n_{1}+2\right)},
$$

respectively. Assume that $m-m_{1}>150$. Then $2^{m-m_{1}}>8 \times\left(8 \times 10^{42}\right)>8 \times\left(n_{1}+2\right)$, therefore

$$
\frac{4}{2^{m-m_{1}} n_{1}}<\frac{1}{2 n_{1}^{2}} \quad \text { and } \quad \frac{4}{2^{m-m_{1}}\left(n_{1}+2\right)}<\frac{1}{2\left(n_{1}+2\right)^{2}} \text {. }
$$

By a criterion of Legendre, it follows that $(m+1) / n_{1}$ or $(m-1) /\left(n_{1}+2\right)$ are convergents of $\tau$, respectively. So, say one of $(m+1) / n_{1}$ or $(m-1) /\left(n_{1}+2\right)$ is of the form $p_{k} / q_{k}$ for some $k=0,1, \ldots, 84$. Here, we use that $q_{84}>8 \times 10^{42}>n_{1}+2$. Then

$$
\frac{1}{\left(a_{k}+2\right) q_{k}^{2}}<\left|\tau-\frac{p_{k}}{q_{k}}\right| \text {. }
$$

Since $\max \left\{a_{k}: k=0, \ldots, 84\right\}=100$, we get

$$
\frac{1}{102 q_{k}^{2}}<\frac{4}{2^{m-m_{1}} q_{k}} \text { and } q_{k} \text { divides one of }\left\{n_{1}, n_{1}+2\right\} .
$$

Thus,

$$
2^{m-m_{1}} \leq 4 \times 102\left(n_{1}+2\right)<4 \times 102 \times 8 \times 10^{42}
$$

giving $m-m_{1} \leq 152$.

Now let us turn to the case that $m-m_{1} \leq 125$ and let us consider inequality (3..8). We put

$$
\Gamma_{2}=n \log \alpha-m_{1} \log 2+\log \left(1 /\left(2 \sqrt{2}\left(2^{m-m_{1}}-1\right)\right)\right),
$$

and we assume that $n-n_{1} \geq 20$. We then have

$$
\left|\Gamma_{2}\right|<\frac{2 \alpha^{5}}{\alpha^{n-n_{1}}} .
$$

Assuming $\Gamma_{2}>0$, we get

$$
0<n\left(\frac{\log \alpha}{\log 2}\right)-m_{1}+\frac{\log \left(\left(1 /\left(2 \sqrt{2}\left(2^{m-m_{1}}-1\right)\right)\right)\right.}{\log \alpha}<\frac{2 \alpha^{5}}{(\log 2) \alpha^{n-n_{1}}}<\frac{237}{\alpha^{n-n_{1}}} .
$$

We apply again Lemma 2.2 with the same $\tau, q, M,(A, B):=(237, \alpha)$ and

$$
\mu_{k}=\frac{\log \left(\left(1 /\left(2 \sqrt{2}\left(2^{k}-1\right)\right)\right)\right.}{\log 2} \quad \text { for } k=1,2, \ldots 125 .
$$

We get $\varepsilon>0.003$, therefore

$$
n-n_{1}<\frac{\log (237 q / 0.003)}{\log \alpha}<137 .
$$

A similar conclusion is reached when $\Gamma_{2}<0$. To conclude, we first get that either $n-n_{1} \leq 131$ or $m-m_{1} \leq 125$. If $n-n_{1} \leq 131$, then $m-m_{1} \leq 171$, and if $m-m_{1} \leq 125$ then $n-n_{1} \leq 136$. In conclusion, we always have $n-n_{1}<137$ and $m-m_{1}<172$. 
Finally we go to (3..10). We put

$$
\Gamma_{3}=n_{1} \log \alpha-m_{1} \log 2+\log \left(\frac{\alpha^{n-n_{1}}-1}{2 \sqrt{2}\left(2^{m-m_{1}}-1\right)}\right) .
$$

Since $n \geq 200$, inequality (3..10) implies that

$$
\left|\Gamma_{3}\right|<\frac{2}{\alpha^{n-4}}=\frac{2 \alpha^{4}}{\alpha^{n}} .
$$

Assume that $\Gamma_{3}>0$. Then

$$
0<n_{1}\left(\frac{\log \alpha}{\log 2}\right)-m_{1}+\frac{\log \left(\left(\alpha^{k}-1\right) /\left(2 \sqrt{2}\left(2^{l}-1\right)\right)\right)}{\log 2}<\frac{2 \alpha^{4}}{(\log 2) \alpha^{n}}<\frac{99}{\alpha^{n}},
$$

where $(k, l):=\left(n-n_{1}, m-m_{1}\right)$. We apply again Lemma 2.2 with the same $\tau, M, q,(A, B):=(99, \alpha)$ and

$$
\mu_{k, l}=\frac{\log \left(\left(\alpha^{k}-1\right) /\left(2 \sqrt{2}\left(2^{l}-1\right)\right)\right)}{\log 2} \quad \text { for } 1 \leq k \leq 137,1 \leq l \leq 171
$$

We have problem at $(k, l)=(1,1),(4,1)$ (as for the case of $\left.\Gamma_{1}\right)$ and additionally for $(k, l)=(8,2)$ since

$$
\frac{\alpha^{8}-1}{2 \sqrt{2}\left(2^{2}-1\right.}=4 \alpha^{4}
$$

We discard the cases $(k, l)=(4,1),(12,1),(8,2)$ for the time being. For the remaining ones, we get $\varepsilon>0.0002$, so we obtain

$$
n \leq \frac{\log (99 q / 0.0002)}{\log \alpha}<139 .
$$

A similar conclusion is reached when $\Gamma_{3}<0$. Hence, $n<150$. Now we look at the cases $(k, l)=$ $(1,1),(4,1),(8,2)$. The cases $(k, l)=(1,1),(4,1)$ can be treated as we did before when we showed that $n-n_{1} \leq 137$ implies $m-m_{1} \leq 171$. The case when $(k, l)=(8,2)$ can be dealt with similarly as well. Namely, it gives

Hence,

$$
\left|\left(n_{1}+4\right) \tau-\left(m_{1}+2\right)\right|<\frac{99}{\alpha^{n}} .
$$

$$
\left|\tau-\frac{m_{1}+2}{n_{1}+4}\right|<\frac{99}{\left(n_{1}+4\right) \alpha} .
$$

Since $n \geq 150$, we have $\alpha^{n}>2 \times 99 \times\left(8 \times 10^{42}\right)>2 \times 99 \times\left(n_{1}+4\right)$, which shows that the right-hand side of inequality (3..11) is at most $2 /\left(n_{1}+4\right)^{2}$. By Legendre's criterion, we get that $\left(m_{1}+2\right) /\left(n_{1}+4\right)=p_{k} / q_{k}$ for some $k \in[0,84]$. We then get by an argument similar to a previous one that

$$
\alpha^{n} \leq 99 \times 102 \times\left(8 \times 10^{42}\right)
$$

giving $n \leq 123$. So, the conclusion is that $n<150$ holds also in the case of the pair $(k, l)=(8,2)$. However, this contradicts our working assumption that $n \geq 150$.

Theorem 1.1 is therefore proved.

\section{Acknowledgements}

We thank the referee for comments which improved the quality of this paper. F. L. was supported in part by grant CPRR160325161141 and an A-rated scientist award both from the NRF of South Africa and by grant no. 17-02804S of the Czech Granting Agency. F.L. worked on this paper during visits at the Mathematics Department to the Purdue University Northwest, USA and the Max Planck Institute for Mathematics, Bonn, Germany in February and March of 2018, respectively. He thanks these institutions for the generous support and hospitality. 


\section{References}

[BaDa69] A. Baker and H. Davenport, The equations $3 x^{2}-2=y^{2}$ and $8 x^{2}-7=z^{2}$, Quart.J of Math. Ser.(2) 20 (1969), $129-137$.

[BrLuYa17] J. J. Bravo, F. Luca, and K. Yazán, On a problem of Pillai with Tribonacci numbers and powers of 2, Bull. Korean Math. Soc. 54.3 (2017), 1069-1080.

[ChPiZi17] K. C. Chim, I. Pink and V. Ziegler, On a variant of Pillai's problem, Int. J. Number Theory 13.7 (2017), $1711-1717$.

[ChPiZi16] K. C. Chim, I. Pink and V. Ziegler, On a variant of Pillai's problem II, J. Number Theory 183 (2018), $269-290$.

[DdLuR17] M. Ddamulira, F. Luca and M. Rakotomalala, On a problem of Pillai with Fibonacci numbers and powers of 2, Proc. Math. Sci. 127.3 (2017), 411-421.

[DdGLu17] M. Ddamulira, C. A. Gómze and F. Luca, On a problem of Pillai with $k$-generalized Fibonacci numbers and powers of 2, (Preprint), 24 pages.

[DuPe98] A. Dujella and A. Pethö, A generalization of a theorem of Baker and Davenport, Quart. J. Math. Oxford Ser. 49.3 (1998), 291-306.

[He35] A. Herschfeld, The equation $2^{x}-3^{y}=d$, Bull. Amer. Math. Soc. 41 (1935), 631.

[He36] A. Herschfeld, The equation $2^{x}-3^{y}=d$, Bull. Amer. Math. Soc. 42 (1936), 231-234.

[Ma00] E. M. Matveev, An explicit lower bound for a homogeneous rational linear form in logarithms of algebraic numbers II, Izv. Math. 64.6 (2000), 1217-1269.

[Mi04] P. Mihăilescu, Primary cyclotomic units and a proof of Catalan's conjecture, J. Reine Angew. Math. 572 (2004), $167-195$.

[Pi36] S. S. Pillai, On $a^{x}+b^{y}=c$, J. Indian Math. Soc. (N.S.) 2 (1936), 119-122.

[Pi37] S. S. Pillai, A correction to the paper On $a^{x}+b^{y}=c$, J. Indian Math. Soc. (N.S.) 2 (1937), 215.

\section{Mohand Ouamar Hernane}

Université des Sciences et de la Technologie Houari-Boumediène (USTHB)

Faculté de Mathématiques, Laboratoire d'Algèbre et Théorie des Nombres, BP 32

16111 Bab-Ezzouar Alger, Algérie

e-mail: mhernane@usthb.dz

\section{Florian Luca}

School of Mathematics, University of the Witwatersrand

Private Bag 3, Wits 2050

Johannesburg, South Africa

AND

Max Planck Institute for Mathematics, Vivatsgasse 7

53111 Bonn, Germany

AND

Department of Mathematics, Faculty of Sciences

University of Ostrava, 30 Dubna 22

70103 Ostrava 1, Czech Republic e-mail: Florian.Luca@wits.ac.za

\section{Salah Eddine Rihane}

Université des Sciences et de la Technologie Houari-Boumediène (USTHB)

Faculté de Mathématiques, Laboratoire d'Algèbre et Théorie des Nombres, BP 32

16111 Bab-Ezzouar Alger, Algérie

e-mail: salahrihane@hotmail.fr

\section{Alain Togbé}

Department of Mathematics, Statistics, and Computer Science

Purdue University Northwest, 1401 S, U.S. 421

Westville IN 46391, USA

e-mail: atogbe@pnw.edu 